\title{
Anabases
}

ANABASES Traditions et réceptions de l'Antiquité

6 | 2007

Varia

\section{Corruption et régénération du politique chez Robespierre}

Thomas Van der Hallen

\section{OpenEdition}

1 Journals

Édition électronique

URL : http://journals.openedition.org/anabases/3448

DOI : 10.4000/anabases.3448

ISSN : 2256-9421

Éditeur

E.R.A.S.M.E.

Édition imprimée

Date de publication : 1 octobre 2007

Pagination : 67-82

ISSN : 1774-4296

\section{Référence électronique}

Thomas Van der Hallen, «Corruption et régénération du politique chez Robespierre », Anabases [En ligne], 6 | 2007, mis en ligne le 01 janvier 2012, consulté le 10 décembre 2020. URL : http:// journals.openedition.org/anabases/3448 ; DOI : https://doi.org/10.4000/anabases.3448

(c) Anabases 
Anabases 6 (2007), p. 67-82.

\section{Corruption et régénération du politique chez Robespierre}

Thomas Van der Hallen

« Dans le système de la Révolution française, [...], ce qui est corrupteur est contre-révolutionnaire " (Robespierre, 17 pluviôse an II).

T Enter d'étudier la pensée politique de Robespierre est, encore de nos jours, dans la France contemporaine, une entreprise délicate, voire périlleuse. En dépit du " consensus républicain ${ }^{1}$ ", l'Incorruptible demeure l'une des figures les plus controversées de l'histoire du pays. Sur un plan à la fois historiographique et politique, sa mémoire oppose, pour faire simple, deux pôles ou plutôt deux camps :

- À main gauche, les derniers tenants de l'historiographie dite "classique " de la Révolution qui, de Louis Blanc à Albert Mathiez et de Jean Jaurès à Albert Soboul, s'est politiquement inscrite dans la tradition du socialisme républicain à la française ; tradition, dans l'ensemble, plutôt favorable à Robespierre en qui elle salue l'apôtre de la démocratie sociale. Témoin, à l'occasion des célébrations du Bicentenaire, cette allocution de Michel Vovelle intitulée Pourquoi nous sommes encore robespierristes 2 ?

- À main droite, les héritiers du courant dit de la "nouvelle histoire ", à l'origine intimement lié à l'apparition de ce qui s'est appelé la "deuxième gauche ", à partir de la fin des années cinquante ${ }^{3}$. Rejoignant pour l'essentiel les thèses du Cold War liberal, ce

1 Par « consensus républicain ", nous entendons simplement le fait que, depuis le second après-guerre, plus aucune force politique parlementaire digne de ce nom ne remet en question la nature républicaine du régime.

2 Cf. M. Vovelle, Combats pour la Révolution française, Paris, La Découverte, 2001, p. 347 359.

3 Le cas de F. Furet, issu de la dissidence du PCF, en offre un bel exemple. 
courant historiographique d'inspiration plutôt tocquevillienne, Jacob Leib Talmon s'est attaché à présenter Robespierre comme l'un des précurseurs du totalitarisme moderne ; sorte de préfiguration du général Jaruzelski, pour reprendre une image popularisée par le cinéaste polonais Andrzej Wajda dans son célèbre film Danton (1982). Selon l'auteur d'une biographie récente, "l'Incorruptible» incarnerait, en effet, "la tentation totalitaire de la gauche française 4 ".

De fait, on ne peut aborder Robespierre sans tenir compte de la persistance de ce débat qui, en dépit du net déclin du courant "classique " depuis le début des années quatre-vingt, n'en demeure pas moins extrêmement passionné. Toutefois, notre intention dans le présent article n'est ni d'analyser la pertinence des arguments des uns et des autres, ni de prendre fait et cause en faveur de l'un ou l'autre camp. Précisons de plus - pour prévenir tout risque de malentendu - que l'objet de notre réflexion n'est pas tant le rôle joué par Robespierre dans l'histoire de la Révolution française que - dans une perspective beaucoup plus théorique - son système de pensée. C'est ce système de pensée que nous nous contenterons de présenter ici - du moins sous l'un de ses aspects - à partir de la fonction qu'y occupe la notion de corruption.

En intitulant notre article corruption et régénération du politique chez Robespierre, nous avons délibérément pris le contre-pied du principe aristotélicien de génération et de corruption, selon lequel tout ce qui naît et croît est, tôt ou tard, condamné à dégénérer, à périr et à pourrir, et qui, appliqué à tout ce qui existe dans le monde sublunaire, induisait une représentation du temps historique, pensé sur le modèle des cycles organiques, dans laquelle jamais rien de nouveau ne pouvait arriver.

À la fin du XVIII' siècle, cette conception classique de l'histoire que la tradition humaniste (et notamment le néo-stoïcisme) avait contribué à relayer, entre en contradiction avec les profonds bouleversements économiques, politiques et sociaux qui marquent les débuts de la révolution industrielle en Angleterre et l'époque des grandes révolutions démocratiques bourgeoises aux Amériques et en Europe. En opposition aux méditations pessimistes et réactionnaires sur la grandeur et la décadence des empires et à l'esthétique des ruines du vieux monde finissant, une idée neuve finit par s'imposer : le progrès, dont la révolution, au sens contemporain de rupture avec un passé périmé, s'avère être l'une des modalités.

Or ce renversement de perspective historique soulève une problématique qui se trouve être au cœur de la pensée politique de Robespierre : comment un nouvel ordre de chose peut-il naître de l'extrême corruption, de la dégénérescence et du pourrissement de l'ancien? 
Pour Robespierre, en effet, l'histoire du politique est l'histoire de sa dégénérescence.

Implicitement, il semble admettre pour point de départ la « maxime fondamentale » du droit politique énoncé par Rousseau dans le Discours sur l'inégalité, à savoir "que les peuples se sont donnés des chefs pour défendre leur liberté et non pour les asservir 5 ". À la tribune de la Constituante, le 31 mai 1791, Robespierre explique, en effet, que si les hommes ont jugé avantageux de se réunir en sociétés politiques, c'est d'abord pour garantir les droits individuels dont ils ne jouissaient à l'état de nature que de façon précaire :

[...] les hommes ont dit : nos forces individuelles sont trop faibles pour protéger notre tranquillité et nos droits; réunissons-les pour en composer une force publique contre laquelle toute force particulière vienne se briser ; réunissons nos volontés pour en former une volonté générale qui, sous le nom de loi, consacre, détermine les droits de chacun ; $[\ldots]^{6}$.

Le politique, à cet égard, repose donc toujours sur l'individualisme bourgeois qui sous-tend toutes les théories du droit naturel moderne. Cependant, dès lors que la "volonté générale ", dont la loi est l'expression, existe, Robespierre affirme que toutes les volontés individuelles ou particulières doivent se subordonner à elle. Aussi, quand le "bien public » ou l'" intérêt général» le requiert, la volonté générale - dont il est l'objet - exige que chaque membre du corps politique soit prêt à sacrifier son "intérêt particulier» : autrement dit, ses biens, sa vie ou encore celle de ses proches. Partant, la fondation du politique passe théoriquement, pour Robespierre comme pour Rousseau, par la transformation de l'homme en citoyen : l'homme naturel individualiste doit désormais devenir l'élément d'une totalité qui l'englobe - la société - et à laquelle il est tenu, le cas échéant, de tout donner, dans la mesure où il n'existe plus que par elle.

Reste à savoir par quel moyen convertir l'entier 1 en unité fractionnaire $1 / \mathrm{n}$. La solution de Robespierre à ce problème réside dans la notion de "vertu " qu'il emprunte à Montesquieu. Principe ou ressort du "gouvernement populaire ", la vertu est une passion politique définie, au livre IV, chapitre V de L'esprit des lois, au sens d'" amour des lois et de la patrie "; "Cet amour, demandant une préférence continuelle de lintérêt public au sien propre, [...] 7 ». Or, dans le Rapport sur les principes de morale politique que Robespierre prononce à la Convention le 17 pluviôse an II, la définition qu'il en donne recoupe presque mot pour mot celle de Montesquieu :

[...] je parle de la vertu publique qui opéra tant de prodiges dans la Grèce et dans Rome, et qui doit en produire de bien plus étonnants dans la France républicaine; de cette 
vertu qui n'est autre chose que l'amour de la patrie et de ses lois. [...] Il est vrai encore que ce sentiment sublime suppose la préférence de l'intérêt public à tous les intérêts particuliers ${ }^{8}$.

À la différence, toutefois, de la vertu chez Montesquieu, conçue comme pur "renoncement à soi-même 9 ", la vertu n'est pour Robespierre que l'identification, par le citoyen, de son intérêt particulier à l'intérêt général ; si bien qu'elle est, en ce sens, bien moins une passion héroïque que la dérivation, dans le politique, de l'amour de soi, voire même de l'amour-propre égoïste.

Bien que la vertu ne soit pas incompatible avec les présupposés individualistes de la pensée de Robespierre, son essor n'entre pas moins, selon lui, en contradiction avec le développement d'une institution, née avec la société elle-même : la propriété. Comme il le dit, le 5 avril 1791, à la Constituante, les progrès de l'inégalité, résultant de l'appropriation abusive de la richesse par un petit nombre d'individus, entraînent la corruption de la morale publique, qui constitue en même temps le premier pas vers la corruption et la dégénérescence du politique :

Quelle vertu, quel bonheur peuvent exister dans un pays où une classe d'individus peut dévorer la substance de plusieurs millions d'hommes? Les grandes richesses enfantent les excès du luxe et des voluptés qui corrompent à la fois, et ceux qui les possèdent et ceux qui les envient ; alors la vertu est méprisée, la richesse seule est un honneur. Les lois ellesmêmes ne sont plus que des instruments entre les mains des riches, pour opprimer les pauvres ; [... ${ }^{10}$.

Or, dès lors que "les lois" sont devenues "des instruments entre les mains des riches", cela signifie que les volontés particulières d'une classe de la société ont été substituées à la volonté générale et que le politique a commencé à dégénérer en un moyen d'oppression.

Dans son discours à la Convention, le 10 mai 1793, Robespierre semble considérer qu'une telle usurpation du pouvoir légitime passe généralement par la confiscation et le détournement de la puissance exécutive du gouvernement au service d'intérêts particuliers :

Le gouvernement est institué pour faire respecter la volonté générale ; mais les hommes qui gouvernent ont une volonté individuelle, et toute volonté cherche à dominer. S'ils emploient à cet usage la force publique dont ils sont armés, le gouvernement n'est plus que le fléau de la liberté ${ }^{11}$. 
Pour Robespierre, le politique dégénère donc, dans un premier temps, au fur et à mesure que la vertu est étouffée sous l'effet des inégalités croissantes et que le gouvernement, confisqué par les riches, tend à usurper la souveraineté. Ces altérations progressives, lorsqu'elles ont atteint un certain degré, se traduisent alors par un changement qualitatif de régime ; ainsi que le suggère ce passage du Rapport du 17 pluviôse:

Une nation est vraiment corrompue, lorsque après avoir perdu, par degrés, son caractère et sa liberté, elle passe de la démocratie à l'aristocratie ou à la monarchie ; c'est la mort du corps politique par la décrépitude ${ }^{12}$.

Par " mort du corps politique ", Robespierre entend, tout comme Rousseau, le moment où l'usurpation de la souveraineté aboutit, par la rupture du pacte social, à un état d'anarchie dans lequel ne subsistent plus entre les hommes que des rapports de force, tels que ceux qui existent entre esclaves et tyrans. De toute évidence, cet état, qui, selon lui, a duré en France "depuis Clovis jusqu'au dernier des Capets ${ }^{13}$ ", est le préalable au despotisme.

Stade ultime de la dégénérescence du politique, le despotisme suit sa propre dialectique de développement. En effet, en avilissant de plus en plus l'espèce humaine, ce régime crée lui-même, selon un rapport de causalité réciproque que Robespierre a parfaitement compris, les conditions morales favorables à l'affermissement de sa domination :

Le despotisme a produit la corruption des mœurs, et la corruption des mœurs a soutenu le despotisme ${ }^{14}$.

Ainsi, pour Robespierre, la "corruption des moeurs", qui, comme nous l'avons montré, est cause de la dégénérescence du politique, en est en même temps l'effet ; et ce pour une raison bien simple. Si être vertueux au sens robespierriste signifie aimer sa patrie, cela suppose d'en avoir une. Or, à partir du moment où la souveraineté a été usurpée par quelques-uns ou par un seul, la masse des citoyens opprimés n'en a plus. Par conséquent, ils ne peuvent pas l'aimer. Telle est, en effet, l'argumentation de Robespierre dans le Rapport du 17 pluviôse:

Dans la monarchie, je ne connais qu'un individu qui peut aimer la patrie, et qui, pour cela, n’a pas même besoin de vertu ; c'est le monarque. La raison en est que de tous les habitants de ses états, le monarque est le seul qui ait une patrie. N'est-il pas le souverain, au moins de fait ? N'est-il pas à la place du peuple ? Et qu'est-ce que la patrie, si ce n'est le pays où l'on est citoyen et membre du souverain ?

12 Robespierre, Euvres [n. 6], t. X, p. 355.

13 Robespierre, Euvres [n. 6], t. IX, p. 496.

14 Robespierre, Euvres [n. 6], t. IX, p. 497. 
Par une conséquence du même principe, dans les États aristocratiques, le mot patrie ne signifie quelque chose que pour les familles patriciennes qui ont envahi la souveraineté ${ }^{15}$.

Négation de la vertu, la "corruption des moeurs", aggravée par le despotisme, réagit sur sa cause pour en accélérer les progrès : c'est elle, en effet, qui encourage les passions et les comportements serviles, pusillanimes et vulgairement égoïstes, si indispensables à son maintien. Dès lors, plus rien ne semble pouvoir enrayer la dynamique d'un tel processus qui, pour ainsi dire, s'autoalimente.

Aussi, comment Robespierre parvient-il à penser la possibilité d'un retournement de l'histoire au sens d'une régénération du politique ? Une Adresse aux Jacobins, publiée dans le huitième numéro du Défenseur de la Constitution, nous fournit un premier élément de réponse :

[...] quand les peuples sont assez éclairés et assez malheureux pour vouloir être libres, ils le sont. Les tyrans tombent d'eux-mêmes, quand ils sont mûrs ${ }^{16}$.

Deux conditions rendant possible le renversement du despotisme sont ici énoncées par Robespierre. En premier lieu, bien sûr, les progrès et la diffusion des lumières de la raison qui constituent, à ses yeux, l'aspect positif du développement de la civilisation humaine et contribuent à préparer l'esprit public, c'est-à-dire la conscience des masses, à la révolution. Mais, il ne suffit pas que "les peuples " soient " assez éclairés"; encore faut-il qu'ils soient " assez malheureux pour vouloir être libres ", condition qui n'est réalisée que quand l'oppression exercée par le despotisme est poussée à son comble. Comme on l'a vu chez Rousseau, le remède est ici dans le mal. Au terme logique de la dégénérescence du politique, "lorsque, dit Robespierre, le 10 mai 1793, l'excès de l'oppression rend [au peuple] son énergie et son indépendance 17 ", le despotisme lui-même engendre une situation critique dont l'une des issues possibles est sa propre négation par la révolution. "Nous touchons au moment qui doit décider à jamais de notre liberté ou de notre servitude, de notre bonheur ou de notre misère 18 ", écrivait déjà, en 1788, le futur député d'Arras, dans sa brochure À la nation artésienne.

Dans la meilleure des hypothèses, la corruption à son degré extrême peut devenir l'amorce d'un processus de régénération que Robespierre pense à la fois comme juste retour des choses et progrès historique. En effet, la révolution tire sa justification du fait qu'elle serait, selon lui, le retour aux véritables principes du droit politique :

Comme l'unique objet de la société est la conservation des droits imprescriptibles de l'homme, le seul motif légitime des révolutions doit être de la ramener vers ce but sacré, et de rétablir ces mêmes droits usurpés par la force et par la tyrannie ${ }^{19}$.

Robespierre, Euvres [n. 6], t. X, p. 353-354.

Robespierre, Euvres [n. 6], t. IV, p. 250.

Robespierre, Euvres [n. 6], t. IX, p. 495.

À la nation artésienne : sur la nécessité de réformer les États d'Artois, édition originale, p. 3. Robespierre, Euvres [n. 6], t. IV, p. 110. 
Pour autant, la révolution n'est nullement la restauration d'un quelconque passé idéalisé, mais au contraire un "pas vers l'avenir ", une phase transitoire vers un ordre de choses supérieur. Selon la formule que Robespierre, dans le Rapport du 18 floréal, emploie pour la définir :

La révolution [...] n'est que le passage du règne du crime à celui de la justice ${ }^{20}$.

Cette transition du mal au mieux n'en est cependant pas moins un processus contradictoire, violent et convulsif, que Robespierre, dans le huitième numéro du Défenseur de la Constitution, compare à un accouchement douloureux :

L'univers est encore dans les douleurs de l'enfantement de la liberté ${ }^{21}$.

En termes moins métaphoriques, la révolution est une guerre : "la guerre de la liberté contre ses ennemis ". Pour Robespierre, en effet, il ne peut en être autrement, à moins de supposer le ralliement improbable de tous à la révolution. Ainsi qu'il l'écrit dans ce passage du troisième numéro du Défenseur de la Constitution:

Si la cour avait pu remplir les premiers serments qu'elle fit à la nation ; si elle avait exécuté loyalement les lois nouvelles, et secondé les progrès de l'esprit public, la révolution était terminée presque aussitôt que commencée par le règne de la paix et de la constitution.

Si la cour, violant ces devoirs sacrés, avait été sans cesse rappelée aux principes de la constitution par la probité incorruptible et par la fermeté inexorable de ceux en qui le peuple semblait avoir mis le plus de confiance, la cause du peuple eût encore facilement triomphé 22 .

Mais pouvait-on attendre de "la cour» qu'elle renonce de bon gré à ses prérogatives ? Quant à l'Assemblée, composée d'hommes nés sous le règne du despotisme, comment aurait-elle pu faire preuve des qualités requises pour être à la hauteur des circonstances ? En effet, la cause de la révolution qui, aux yeux de Robespierre, coïncide avec le bien public et l'intérêt général de l'humanité, exige de la vertu de la part de chacun :

Le devoir de tout homme et de tout citoyen est [...] de concourir, autant qu'il est en lui, au succès de cette sublime entreprise, en sacrifiant son intérêt particulier à l'intérêt général 23 . 
Or, comme il le constate à la tribune des Jacobins, le 28 octobre 1792, "les hommes de la révolution étaient les hommes de l'ancien régime 24 ", avec tout ce que cela implique, selon lui, de vices, de mauvaises habitudes et de préjugés d'éducation.

Se pose alors à Robespierre le problème suivant : si le despotisme a corrompu les hommes, quel est, dans la société présente, la force susceptible de mener la révolution à bon terme?

[...] quelle est la puissance qui opérera ce prodige ? Je m'effraye, si ceux qui ont vieilli sous le régime d'une société corrompue, sont ceux qui prétendent régénérer les mœurs publiques. Suivant le cours naturel des choses, nos neveux sont destinés à être meilleurs que nous; et c'est nous qui devons les instituer 25.

Pour Robespierre, la solution à ce problème réside, non pas dans les individus, mais dans la masse ou, plus exactement encore, dans le "peuple ", tel qu'il le définit dans un discours à la Convention, le 28 décembre 1792 :

[...] il faut entendre par le peuple, la nation, moins les ci-devant privilégiés et les honnêtes gens $;[\ldots]^{26}$.

Sorte de nation-classe pour ainsi dire, le peuple, au sens de Robespierre, est le seul véritable sujet de la révolution, la seule force positivement révolutionnaire ; ce qu'il l'affirme dès 1790, dans son Discours sur l'organisation des gardes nationales:

[...] qui a fait notre glorieuse révolution ? Sont-ce les riches ? sont-ce les hommes puissants ? Le peuple seul pouvait la désirer et la faire ; le peuple seul peut la soutenir, par la même raison $27 \ldots$

Le peuple seul veut sincèrement la révolution, précisément parce qu'étant, par définition, la nation à l'exclusion des classes parasitaires de la société, son intérêt propre est supposé identique à l'intérêt général. "Le peuple est toujours pur dans ses motifs ", aime à répéter Robespierre, "il ne peut aimer que le bien public, puisque le bien public n'est que l'intérêt du peuple 28 ». Or, puisque le but de la révolution est conforme au "bien public ", il se confond nécessairement aussi avec "l'intérêt du peuple "; donc le peuple est révolutionnaire.

La force de ce raisonnement tient au fait qu'il ne présuppose même pas que le peuple, ni que les individus qui le composent, soient vertueux ; ce qui, à l'évidence, serait en contradiction avec l'idée que le despotisme a corrompu les hommes. Comme l'explique, en effet, Robespierre, dans une Lettre à ses commettants du mois de janvier 1793 : 
Le peuple veut toujours le bien public, parce qu'il est le peuple ; il n'a pas même besoin de vertu, pour être juste ; car c'est à lui-même qu'il rend justice : [...] ${ }^{29}$.

N'ayant "pas même besoin de vertu ", le peuple est donc politiquement plus frable que les individus qui, eux, au contraire, n'en ont jamais de trop. D'où le principe général, posé par Robespierre, selon lequel « le peuple vaut toujours mieux que les individus 30 ".

À la différence du peuple, en effet, les individus ont, sous l'influence corruptrice du despotisme, pris l'habitude de préférer leur intérêt particulier à l'intérêt général. Or de cette préférence procède inévitablement la division du corps politique en différents partis ou factions, se détachant de la masse du peuple. "Qu'est-ce qui forme plusieurs partis dans une société quelconque ? ", demande Robespierre aux Jacobins, le 22 février 1792 : "C'est l'intérêt seul, c'est l'intérêt personnel. L'intérêt général est gravé également dans le cœur de tous les hommes, il n'y a que l'intérêt personnel, il n'y a que l'intrigue et l'ambition qui les divisent et je vous défie de citer un homme attaché invariablement à la liberté, qui n'ait marché constamment sur la ligne directe ${ }^{31}$. »

En temps de révolution, les individus dont l'intérêt particulier entre en conflit avec l'intérêt général sont principalement de deux sortes :

1. Ceux que l'abolition de l'ancien ordre de choses a lésé dans leurs privilèges, dans leurs possessions ou dans leurs revenus. En d'autres termes, il s'agit, comme le dit Robespierre, de "cette poignée de citoyens égarés par l'orgueil, que nous avons honorés du nom d'aristocrates 32 ";

2. Ceux qui ne voient, dans la révolution, que l'occasion de monter à la fortune et au pouvoir et ambitionnent de devenir les privilégiés du nouveau régime. Plus dangereux encore que les " francs aristocrates" parce que plus hypocrites, ces derniers forment la catégorie que Robespierre désigne généralement sous les noms de "trầtres ", d' "intrigants" ou de "faux patriotes ». De leur coalition naissent les différentes " factions " qui accompagnent la marche du mouvement révolutionnaire aussi longtemps que les intérêts particuliers qu'elles représentent s'accordent avec l'intérêt du peuple. Après quoi, elles se séparent de lui, rejoignant ainsi les aristocrates dans le camp de la contre-révolution, selon un processus dont Robespierre, dans une Lettre à ses commettants du mois de septembre 1792, décrit très bien le caractère dialectique :

Avant l'abolition de la noblesse et de la royauté, les intrigants, qui ne songeaient qu'à élever leur fortune sur les ruines de la cour, combattaient à côté des amis de la liberté, et partageaient, avec eux, le titre de patriotes. De là, les diverses métamorphoses de tant de personnages, dont la vertu civique expirait, au moment où elle commençait à contrarier

Robespierre, Euvres [n. 6], t. V, p. 200.

Robespierre, Euvres [n. 6], t. V, p. 209.

Robespierre, Euvres [n. 6], t. VIII, p. 202.

Robespierre, Euvres [n. 6], t. VIII, p. 181. 
leurs spéculations ambitieuses. Alors la nation semblait divisée en deux parties, les royalistes et les partisans de la cause populaire. Aujourd'hui que l'ennemi commun est terrassé, vous verrez ceux que l'on confondait sous le nom de patriotes se diviser nécessairement en deux classes. Les uns voudront constituer la république pour eux-mêmes, et les autres pour le peuple, suivant la nature des motifs qui avait jusque-là excité leur zèle révolutionnaire. Les premiers s'appliqueront à modifier la forme du gouvernement, suivant les principes aristocratiques et l'intérêt des riches [...] : les autres chercheront à la fonder sur les principes de l'égalité et sur l'intérêt général 33 .

Ainsi, à chaque étape de la révolution, la préférence de l'intérêt particulier chez certains divise le camp révolutionnaire en deux. Et, en ce sens, on peut dire que, dans la compréhension que Robespierre a de la révolution en tant que phénomène, la corruption, héritage maudit de l'ancien régime, fait office de principe de négativité. En effet, dans la mesure où elle met en contradiction les intérêts particuliers avec l'intérêt général, c'est elle qui cause et renouvelle sans cesse au sein du corps politique l'antagonisme entre d'une part, le peuple et, d'autre part, les ennemis du peuple.

"Il n'y a plus que deux partis en France, le peuple et ses ennemis 34 », déclare Robespierre, le 8 mai 1793 ; les « ennemis » du peuple étant précisément, selon lui, « les hommes corrompus qui préferent leur intérêt à l'intérêt général 35 ".

Or, entre "le peuple et ses ennemis ", il n'existe ni conciliation, ni tiers parti : «Celui qui n'est pas pour le peuple est contre le peuple [... ${ }^{36}$. " Partant, la lutte est inévitable. Bien loin, cependant, d'être stérile, cette lutte est, aux yeux de Robespierre, le principe moteur de la dynamique révolutionnaire qu'il conçoit, dans le Rapport du 27 brumaire an II, sur le modèle physique de l'action et de la réaction :

Les temps qui devaient enfanter le plus grand des prodiges de la raison devaient aussi être souillés par les derniers excès de la corruption humaine. Les crimes de la tyrannie accélérèrent les progrès de la liberté, et les progrès de la liberté multiplièrent les crimes de la tyrannie, en redoublant ses alarmes et ses fureurs. Il y a eu, entre le peuple et ses ennemis, une réaction continuelle dont la violence progressive a opéré en peu d'années l'ouvrage de plusieurs siècles 37 .

Toute action entraînant une réaction en sens contraire, les menées contre-révolutionnaires des ennemis $d u$ peuple suscitent la réaction du peuple. Mais cette réaction étant, en même temps, une action, non seulement elle précipite le mouvement révolu-

Robespierre, Euvres [n. 6], t. V, p. 17.

Robespierre, Euvres [n. 6], t. IX, p. 487.

Robespierre, Euvres [n. 6], t. IX, p. 487.

Robespierre, Euvres [n. 6], t. IX, p. 488.

Robespierre, Euvres [n. 6], t. X, p. 168. 
tionnaire en avant, mais elle provoque à son tour la réaction des forces de la contrerévolution et ainsi de suite.

De par le caractère contradictoire de sa logique processuelle, la révolution ne progresse donc pas de manière régulière, graduelle et continue, mais passe, à l'inverse, par une succession de phases critiques d'accélération et de rétrogradation. En effet, comme l'observe très justement Robespierre, dans son Discours contre la guerre du 2 janvier 1792:

Il est dans les révolutions des mouvements contraires et des mouvements favorables à la liberté, comme il est dans les maladies des crises salutaires et des crises mortelles 38 .

Ainsi qu'il le précise lui-même aussitôt : "Les mouvements favorables sont ceux qui sont dirigés contre les tyrans, comme l'insurrection des Américains, ou comme celle du 14 juillet ${ }^{39}$. " Autrement dit, il s'agit de l'action révolutionnaire du peuple. En revanche, les «mouvements contraires » ou «à contresens » sont, selon lui, l'effet de la réaction contre-révolutionnaire des ennemis du peuple. Dans une sorte de "profession de foi " politique, publiée aux pires heures de l'été 1791 - peu de temps après la fusillade du Champ-de-Mars -, Robespierre affirme avoir très tôt compris que "si des ambitieux, étrangers par leur caractère et leur éducation au sentiment de l'égalité et à l'amour du peuple ", c'est-à-dire des hommes corrompus sous l'ancien régime, " osaient se déclarer les chefs de la révolution, pour la diriger vers leur but particulier », le mouvement révolutionnaire rétrograderait "de faiblesse en faiblesse, et d'erreur en erreur, à un état à peu près tel que le premier 40 ». Cet " état " - dont le réveil des anciennes habitudes et des vieux préjugés, encouragé par les forces corruptrices de la contre-révolution, a bien évidemment favorisé le retour sous des formes nouvelles - est celui que dépeint Robespierre, dans le discours précité du 2 janvier 1792 :

Le moment arrive où la division règne partout, où tous les pièges des tyrans sont tendus, où la ligue de tous les ennemis de l'égalité est entièrement formée, où les dépositaires de l'autorité publique en sont les chefs, où la portion des citoyens qui a le plus d'influence par ses lumières et par sa fortune est prête à se ranger de leur parti. Voilà la nation placée entre la servitude et la guerre civile 41 .

Lissue de cette phase de reflux de la révolution, lorsqu'elle a atteint son point ultime, se résume alors, de nouveau, à l'alternative entre "servitude " et "guerre civile» : soit la restauration plus ou moins intégrale de l'ancien despotisme, soit une réaction des masses en sens contraire.

Robespierre, Euvres [n. 6], t. VIII, p. 86. Robespierre, Euvres [n. 6], t. VIII, p. 86. Robespierre, Adresse aux Français, in Robespierre, Écrits présentés par Claude Mazauric, Messidor/Éditions sociales, Paris, 1989, p. 126.

RobesPIERRE, Euvres [n. 6], t. VIII, p. 91. 
C'est cette dernière éventualité que Robespierre semble appeler de ses vœux, en juin 1792, dans un article du Défenseur de la Constitution où il écrit :

[...] le nombre des intrigants est infini ; leur corruption est extrême ; la fureur et la perfidie des tyrans sont sans bornes; mais le peuple est bon, la cause de l'humanité est sainte, et le ciel est juste. De l'excès de nos maux naitra le remède 42 .

Linsurrection du 10 août, qui, quelques semaines plus tard, balaye à la fois la monarchie et le compromis constitutionnel de 1791, est venue confirmer la prédiction de Robespierre. Aussi, dans l'Adresse qu'il présente, le $1^{\text {er }}$ septembre, au nom du Conseil général de la Commune, réaffirme-t-il le principe selon lequel le remède est dans l'excès du mal:

Qu'étions-nous, avant la journée du 10 août 1792 ? les esclaves et les jouets de tous les mandataires du peuple, des victimes vouées aux vengeances de la cour. [...]. L'excès du mal produisit encore une fois le remède ; et le peuple se leva, il fut forcé d'acheter la victoire par le sang des meilleurs citoyens 43 .

De par sa portée politique, le 10 août n'est cependant pas la simple réitération du 14 juillet, mais semble bien plutôt marquer, aux yeux de Robespierre, une étape supérieure du processus révolutionnaire, comme l'indique ce passage du Défenseur de la Constitution, dans lequel il compare les deux événements :

[...] le peuple a reparu dans l'attitude qu'il avait montrée au mois de juillet 1789.

Seulement il est vrai, que l'insurrection du 10 août 1792, a sur celle du 14 juillet 1789 , des avantages qui annoncent le progrès des lumières, depuis cette première époque de la révolution.

En 1789, le peuple de Paris se leva tumultuairement, pour repousser les attaques de la cour, pour s'affranchir de l'ancien despotisme, plutôt que pour conquérir la liberté, dont l'idée était encore confuse et les principes inconnus. Toutes les passions concoururent alors à l'insurrection dont il donna le signal à la France entière.

En 1792, il s'est levé avec un sang-froid imposant, pour venger les lois fondamentales de sa liberté violée, pour faire rentrer dans le devoir tous les tyrans qui conspiraient contre lui, tous les mandataires infidèles qui cherchaient à ensevelir encore une fois les droits imprescriptibles de l'humanité. Il a exécuté les principes proclamés trois ans auparavant, par ses premiers représentants ; il a exercé sa souveraineté reconnue, et déployé sa puissance et sa justice, pour assurer son salut et son bonheur.

En 1789, il était aidé par un grand nombre de ceux que l'on appelait grands, par une partie des hommes qui étaient revêtus de la puissance du gouvernement.

En 1792, il a trouvé toutes ses ressources, et dans ses lumières, et dans sa force; seul, il a protégé la justice, l'égalité et la raison, contre tous leurs ennemis. Ce n'était point 
seulement le peuple de Paris qui donnait un grand exemple à la France, c'était le peuple français qui se levait à la fois 44 .

Linsurrection du 10 août 1792, qui semble reproduire celle du 14 juillet 1789 à un stade plus élevé, constitue donc un progrès par rapport à elle. Toutefois, Robespierre a conscience que ce passage du " moins bien " au " mieux " n'a pu s'effectuer que par la médiation du " pire ", du négatif porté à son comble ; c'est-à-dire, selon ses propres termes, des "derniers excès de la corruption humaine 45 ". Aussi, les ennemis du peuple, ces « hommes corrompus" qui, par intérêt particulier, tentent d'arrêter la révolution, ne font-ils en réalité que la servir à leur insu : sans eux, la marche du processus révolutionnaire n'aurait pu aller aussi loin. C'est, en effet, ce que montre Robespierre, dans le Rapport du 27 brumaire, à propos du rôle contradictoire joué par le ministre anglais William Pitt, en qui il voit le chef de la contre-révolution :

Voyez comme chaque crise de notre Révolution l'entraîne toujours au-delà du point où il voulait l'arrêter ; [...]. À la fin de 1791, il croyait préparer insensiblement la chute du roi Capet, en conservant le trône pour le fils de son maître ; mais le 10 août a lui, et la République est fondée. C'est en vain que, pour l'étouffer dans son berceau, la faction girondine et tous les lâches émissaires des tyrans étrangers appellent de toutes parts les serpents de la calomnie, le démon de la guerre civile, l'hydre du fédéralisme, le monstre de l'aristocratie : le 31 mai, le peuple s'éveille, et les traîtres ne sont plus 46.

Ainsi, le cours de la révolution, qui, comme le voit très bien Robespierre dans ce texte, tire en partie sa force de l'opposition même qu'il suscite, ne progresse pas, selon lui, en ligne droite, mais plutôt en spirale ou plus exactement de manière hélicoïdale (cf. Fig. 1).

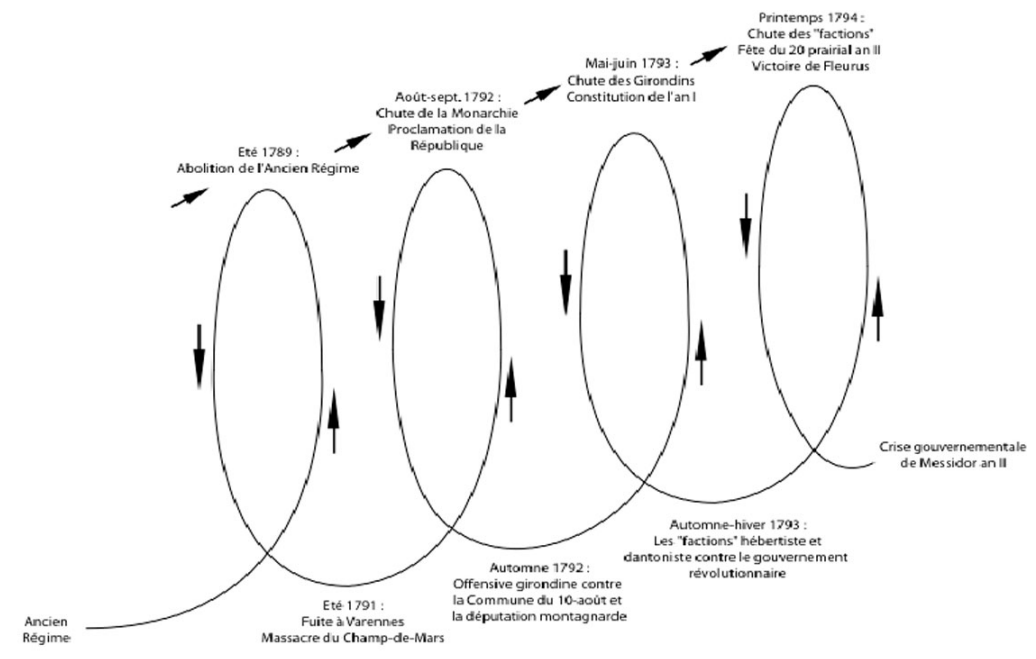

Figure 1.

Représentation schématique des processus révolutionnaires 
En conclusion de cet article, il convient d'abord de récapituler synthétiquement en quoi réside l'importance que revêt la notion de corruption dans la pensée de Robespierre.

1. La corruption est, pour lui, la négation de la vertu. Elle consiste dans la préférence que l'individu accorde à son intérêt particulier au détriment de l'intérêt général.

2. En ce sens politico-moral, la corruption est la cause - et réciproquement l'effet - de la corruption du corps politique, c'est-à-dire de son processus historique de dégénérescence.

3. C'est cependant la corruption du politique à son degré le plus extrême (sous la forme du despotisme), qui crée elle-même les conditions d'une révolution régénératrice.

4. La corruption, au sens politico-moral, ne disparaît pourtant pas instantanément avec ce retournement de l'histoire, mais subsiste comme héritage de l'ancienne société et devient le principe de négativité d'où procède la contradiction entre la révolution et la contre-révolution.

5. À ce titre, la corruption joue un rôle moteur dans le processus révolutionnaire de régénération du politique.

Par une conséquence inévitable des points $4^{\circ}$ ) et $5^{\circ}$ ), Robespierre pensait que la révolution ne pouvait se terminer qu'avec, pour ainsi dire, la négation du négatif, c'està-dire l'extirpation de la corruption. De là le double « ressort du gouvernement populaire en révolution ", tel qu'il le théorise dans ses Rapports des 5 nivôse et 17 pluviôse an II : « à la fois la vertu et la terreur 47 ». En effet, pour accélérer la régénération du politique, la tâche du pouvoir révolutionnaire est, d'une part, de régénérer les mœurs par la mise en œuvre d'un vaste plan d'éducation publique, ayant pour but d'éveiller en chacun le sentiment de la vertu. Comme le déclare Robespierre, à la Convention, le 17 pluviôse, « tout ce qui tend à exciter l'amour de la patrie, [...], à diriger les passions du cœur humain vers l'intérêt public, doit être adopté ou établi par vous. Tout ce qui tend à les concentrer dans l'abjection du moi personnel, [...], doit être rejeté ou réprimé par vous 48 ". La contrepartie de cette politique éducative est la terreur par laquelle les comités de la Convention exercent, en quelque sorte, la dictature de l'intérêt général et brisent la résistance contre-révolutionnaire de l'intérêt particulier : " Le gouvernement révolutionnaire ", affirme-t-il dans le Rapport du 5 nivôse, "ne doit aux ennemis du peuple que la mort 49 ".

Sur le plan théorique, on peut dire que le grand mérite de Robespierre est d'avoir su formuler, selon l'expression de G. Labica, la « première systématisation 50 » du phénomène révolutionnaire et d'en avoir mieux perçu le caractère dialectique que la 
plupart de ses contemporains. En effet, ce qu'il a particulièrement bien compris, c'est que le ressort qui fait avancer l'histoire - et la révolution, en tant que modalité du progrès historique -, c'est la contradiction, la lutte entre des forces contraires. Comme il le rappelle encore dans son dernier discours, le 8 thermidor an II, la "République ", qui représente, à ses yeux, une étape supérieure à la monarchie constitutionnelle dans la marche du processus révolutionnaire, a été " amenée par la force des choses et par la lutte des amis de la liberté contre des conspirations toujours renaissantes 51 ». Si cette compréhension profonde du rôle moteur de la conflictualité dans la logique des événements explique, dans une certaine mesure, l'intelligence tactique qui a permis à Robespierre d'investir le pouvoir révolutionnaire et de réussir à l'exercer pendant presque un an, sa pensée n'en a cependant pas moins le défaut de réduire le principe dynamique de la révolution à une simple contradiction d'ordre politico-moral. «Toutes nos querelles, écrit-il dans le numéro 4 du Défenseur de la Constitution, ne sont que la lutte des intérêts privés contre l'intérêt général, de la cupidité et de l'ambition contre la justice et l'humanité 52 .» Or, derrière ce qu'il appelle « lutte des intérêts privés contre l'intérêt général ", Robespierre relègue au second plan les luttes de classes et leurs enjeux réels; ce qui l'empêche d'avoir une conscience aussi nette du sens historico-social de la Révolution que certains de ses adversaires politiques, tels Mirabeau, Sieyès ou Barnave. Ainsi, par exemple, la clairvoyante intuition de ce dernier pour qui l'événement révolutionnaire français serait une "nouvelle distribution du pouvoir » - corrélat d'une " nouvelle distribution de la richesse " - au profit d'une "sorte d'aristocratie bourgeoise et marchande ", détentrice de "la propriété industrielle et mobilière 53 ", lui demeure foncièrement étrangère. Ou s'il lui arrive également d'entrevoir le caractère "bourgeois » de la Révolution, c'est pour le dénoncer, mais jamais - comme chez Barnave pour en démontrer la nécessité historique. Robespierre, en effet, croyait sérieusement en la vérité des principes rationnels abstraits énoncés par les théoriciens du droit naturel dans leur acception démocratique la moins restrictive; et quand il affirme, dans le Rapport du 18 floréal, vouloir «mettre dans les lois et dans l'administration les vérités morales reléguées dans les livres des philosophes 54 ", sa sincérité ne fait aucun doute. Aussi, contrairement à Barnave, l'égoïsme de classe de ce que lui aussi désigne par l'expression d'" aristocratie bourgeoise 55 " ne pouvait être, à ses yeux, qu'un obstacle irréductible à la révolution. Rien ne le révèle plus clairement que le raisonnement qu'il tient dans des notes personnelles retrouvées dans ses papiers après sa mort :

51 RobesPierre, Euvres [n. 6], t. X, p. 545 (souligné par nous). 
Quel est le but ? L'exécution de la constitution en faveur du peuple. Quels seront nos ennemis ? Les hommes vicieux et les riches. Quels moyens emploieront-ils ? La calomnie et l'hypocrisie. Quelles causes peuvent favoriser l'emploi de ces moyens ? L'ignorance des sans-culottes. Il faut donc éclairer le peuple. [...] Quand le peuple sera-t-il donc éclairé ? Quand il aura du pain, et que les riches et le gouvernement cesseront de soudoyer des plumes et des langues perfides pour le tromper ; lorsque leur intérêt sera confondu avec celui du peuple. Quand leur intérêt sera-t-il confondu avec celui du peuple ? JAMAIS 56.

L'échec politique de Robespierre est inscrit en toutes lettres dans ce "JAMAIS ». Ce n'est, pourtant, qu'à la veille de sa chute qu'il semble enfin pleinement réaliser l'incompatibilité entre le sens objectif du mouvement révolutionnaire et l'idéal politique abstrait qu'il croyait subjectivement en être le «but», lorsque il déclare tragiquement, dans son dernier discours à la Convention :

Ma raison, non mon cœur, est sur le point de douter de cette république vertueuse dont je m’étais tracé le plan 57.

De fait, son rêve de "république vertueuse ", dans laquelle le bonheur de chacun se serait confondu dans le bonheur de tous, était impossible sur la base de la société bourgeoise réelle qui était en train de naître ; car la propriété privée, les rapports sociaux fondés sur le salaire - choses que lui-même ne remettait pas en cause -, ainsi que le principe même de la concurrence, ne pouvaient que renouveler indéfiniment la contradiction entre l'intérêt particulier et l'idée d'intérêt général, rendue de toute façon fictive par l'antagonisme croissant entre travail salarié et capital. Quel intérêt général à proprement parler peut-il, en effet, y avoir, tant que la société reste une société divisée en classes, dans laquelle la condition de possibilité du profit pour la classe qui détient et gère les moyens de production réside, en dernière analyse, dans l'extorsion de la plusvalue créée par la classe qui vend sa force de travail contre un salaire ; en un mot, tant qu'il existe des exploiteurs et des exploités ? Partant, la tentative robespierriste de résoudre cette contradiction entre intérêts particuliers et intérêt général, dans le cadre des rapports bourgeois, était vaine et, en ce sens, ne pouvait signifier que le recours sans fin au couperet de la guillotine.

Thomas VAN DER HALLEN
5,rue de Capri
75012 Paris
van.der.hallen@aliceadol.fr

56 E.-B. CourToIs, Papiers inédits trouvés chez Robespierre, Saint-Just, Payan etc., précédé du Rapport fait au nom de la commission chargée de l'examen des papiers de Robespierre, t. II, p. 13-14. 